\title{
REFINEMENTS OF CAUCHY-SCHWARZ NORM INEQUALITY
}

\author{
JUNMIN HAN AND JIAN SHI
}

Abstract. In this paper, we utilize the convexity of the function $f(v)=\left\|\left|A^{v} X B^{1-v}\right|^{r}\right\| \cdot\left\|\left|A^{1-v} X B^{v}\right|^{r}\right\|$ and the Hermite-Hadamard inequality to obtain a family of new refinements of Cauchy-Schwarz norm inequality for operators, which extends the related results.

Mathematics subject classification (2010): 47A63.

Keywords and phrases: Cauchy-Schwarz norm inequality, Hermite-Hadamard inequality, Heinz inequality, convex functions.

\section{REFERENCES}

[1] H. AbBas And B. Mourad, A family of refinements of Heinz inequalities of matrices, J. Inequal. Appl. 1 (2014), 1-7.

[2] R. Bhatia, Matrix analysis, Springer-Verlag, New York, 1997.

[3] R. BhatiA AND C. DAvis, A Cauchy-Schwarz inequality for operators with applications, Linear Algebra Appl. 223-224 (1995), 119-129.

[4] P. S. Bullen, A Dictionary of Inequalities, Pitman Monographs and Surveys in Pure and Applied Mathematics, vol. 97, Longman, Harlow (1998).

[5] A. BURQAN, Improved Cauchy-Schwarz norm inequality for operators, Journal Math. Inequal. 10 (2016), 205-211.

[6] Y. Feng, Refinements of the Heinz inequalities, J. Inequal. Appl. 18 (2012), 1-6.

[7] F. HIAI AND X. ZHAN, Inequalities involving unitarily invariant norms and operator monotone functions, Linear Algebra Appl. 341 (2002), 151-169.

[8] R. HORN AND R. MATHIAS, Cauchy-Schwarz inequalities associated with positive semidefinite matrices, Linear Algebra Appl. 142 (1990), 63-82.

[9] S. WANG, Some new refinements of Heinz inequalities for matrices, J. Inequal. Appl. 1 (2013), 132136.

[10] Y. Yan, Y. Feng, G. Chen, Refinements of the Heinz inequalities for matrices, J. Inequal. Appl. 50 (2014), 1-6. 